\title{
Insulin glargine and insulin have identical effects on proliferation and phosphatidylinositol 3-kinase/AKT signalling in rat thyrocytes and human follicular thyroid cancer cells
}

\author{
K. Müller • C. Weidinger • D. Führer
}

Received: 5 November 2009 / Accepted: 21 December 2009 / Published online: 24 February 2010

(C) Springer-Verlag 2010

Keywords Insulin glargine $\cdot$ Phosphatidylinositol

3-kinase/AKT cascade $\cdot$ Proliferation $\cdot$ Thyroid cancer

\author{
Abbreviations \\ FOXO Forkhead box $\mathrm{O}$ \\ FRTL-5 Fisher rat thyroid low serum 5\% (cells) \\ PTEN Phosphatase and tensin homologue
}

To the Editor: The mitogenic potency of the analogous insulin glargine (A21Gly,B31Arg,B32Arg human insulin) is currently under heated debate [1], since several clinical studies have raised the possibility of insulin glargine treatment having a carcinogenic potential, especially in breast cancer [2]. Moreover, latest data from Shukla et al. [3] suggest that insulin glargine may indeed trigger cellular proliferation in MCF-7 breast cancer cells at lower dosages than normal insulin.

In the past years, the insulin/phosphatidylinositol 3-kinase (PI3K)/protein kinase B (AKT) signalling cascade has emerged as a central mediator of thyroid carcinogenesis [4]. Our group recently observed that PI3K/AKT mediated inactivation of the tumour suppressor forkhead box $\mathrm{O}$ (FOXO) 3 is a hallmark of follicular and papillary thyroid carcinogenesis [5]. Physiologically, FOXO3 accumulates in

K. Müller and C. Weidinger contributed equally to this study.

K. Müller $\cdot$ C. Weidinger $\cdot$ D. Führer $(\triangle)$

Department of Internal Medicine,

Division of Endocrinology and Nephrology, University of Leipzig,

Liebigstr. 18,

04103 Leipzig, Germany

e-mail: dagmar.fuehrer@medizin.uni-leipzig.de the nucleus, where it controls the transcription of antiproliferative and pro-apoptotic targets such as growth arrest and DNA-damage-inducible-protein 45 (GADD45), cylindependent kinase inhibitor $1 \mathrm{~B}\left(\mathrm{p} 27^{\mathrm{kip} 1}\right)$ and BCL2-like 11 (BIM). In response to $\mathrm{PI} 3 \mathrm{~K} / \mathrm{AKT}$ activation, FOXO3 is phosphorylated and subsequently translocates to the cytoplasma, where it is proteosomally degraded [6]. Furthermore, loss of PTEN, a crucial inhibitor of PI3K activity, results in the post-translational inactivation of FOXO3 in murine thyroids and in tumour progression in a mouse model of thyroid follicular carcinogenesis [7].

Since few data on the mitogenic potency of insulin glargine exist for the thyroid, we wondered how insulin glargine influenced cellular proliferation in normal and malignant thyroid cells, and to what extent the activity of the tumour suppressor FOXO3 might be impaired by insulin glargine treatment, when compared with stimulation by normal insulin. We therefore investigated the proliferative impact of insulin glargine in epithelial rat thyrocytes (Fisher rat thyroid low serum 5\% [cells]; FRTL-5), which are highly sensitive to insulin stimulation, which results in inhibition of FOXO3 and induction of cellular proliferation [5]. We also sought to uncover possible distinct influences of insulin glargine vs normal insulin on the proliferative properties of thyroid cancer cells with inherent overactivation of the insulin/PI3K/AKT signalling axis. To this aim, we studied the human follicular thyroid cancer cell line FTC-133, which exhibits low abundance of the PI3K inhibitor PTEN [8].

Incubating cells with different amounts of normal insulin or insulin glargine, we observed that stimulation with both types of insulin (range $1 \mathrm{ng}$ to $10 \mu \mathrm{g}$ ) increased proliferation of FRTL-5 cells when compared with controls kept in insulin-free media (Fig. 1a). Treatment with insulin glargine did not significantly augment the number of cells in 
Fig. 1 Impact of normal insulin vs insulin glargine on (a) proliferation of thyroid epithelial cells (rat FRTL-5). Cell numbers were quantified by cell counting on days 2, 4 and 6 using a Neubauer counting chamber (Carl Roth, Karlsruhe, Germany) (plating $1 \times 10^{4}$ cells in 12-well plates). The insulin concentrations were adapted on their specific insulin molarity. Continuous line, normal insulin; dashed line, insulin glargine; black squares, control; upright triangles, $1 \mathrm{ng}$; inverted triangles, $10 \mathrm{ng}$; black diamonds, $100 \mathrm{ng}$; black circles, $1 \mu \mathrm{g}$; white squares, $10 \mu \mathrm{g}$. b Induction of pro-apoptotic, anti-proliferative $\mathrm{FOXO} 3$ target genes Gadd45, (c) $p 27^{k i p l}$ and (d) Bim, fold changes compared with control cells (Ctr) kept in insulin-free medium. mRNA expression determined by real-time PCR as previously described [5]. Normal insulin, black bars; insulin glargine, grey bars. e Activation of PI3K/AKT signalling in rat FRTL-5 cells and human follicular thyroid cancer cells (data not shown) with endogenous loss of PTEN. $\mathrm{PI} 3 \mathrm{~K} / \mathrm{AKT}$ activation was assessed by Western blot using specific antibodies against phosphorylated (p)AKT, pFOXO3A, FOXO3A and $B$-actin as previously described [5]. NI, normal insulin a

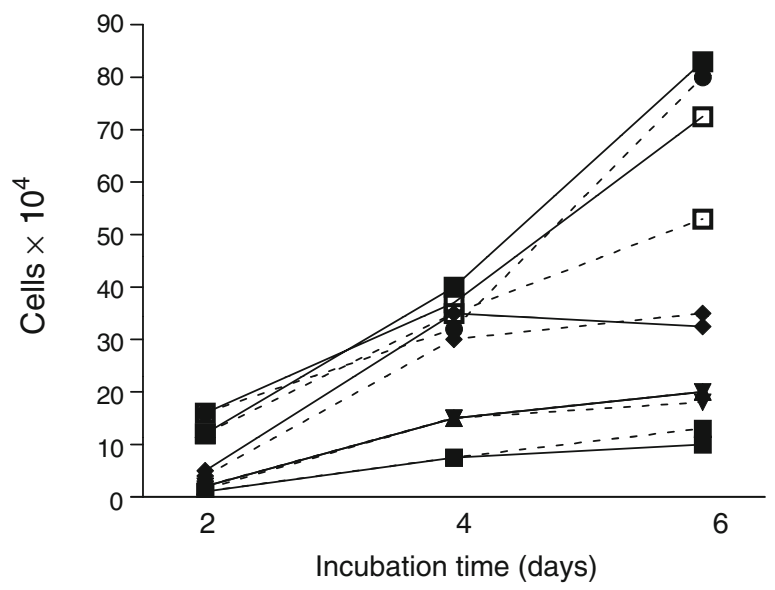

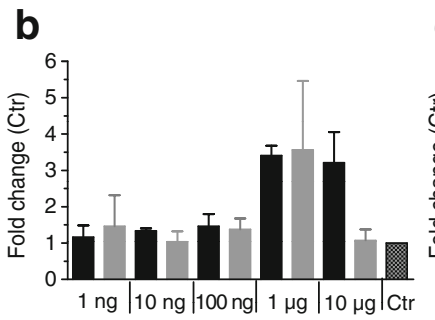

c

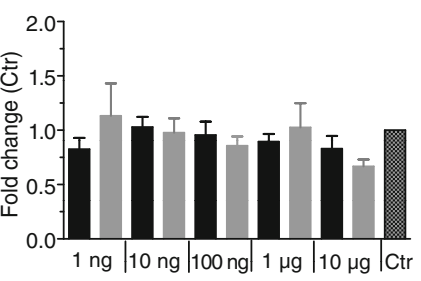

d

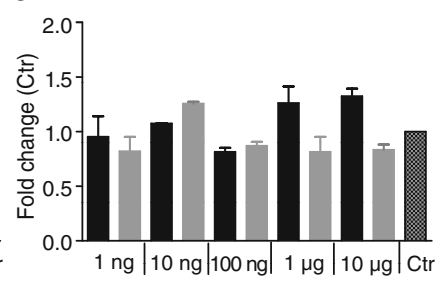

e

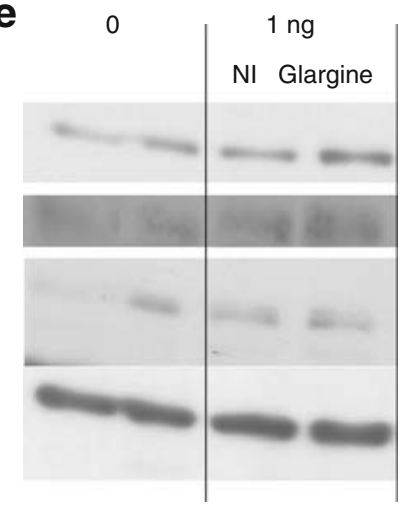

$10 \mathrm{ng} \quad 100 \mathrm{ng} \quad 1 \mu \mathrm{g}$ comparison to samples stimulated with normal insulin (Fig. 1a). Notably, proliferation of FTC-133 cancer cells was not affected by normal insulin or by the insulin analogue, glargine (Fig. 1a), suggesting that loss of endogenous PTEN in these cells already results in maximal activation of PI3K/AKT even under insulin-free conditions.

To uncover the impact of insulin glargine vs normal insulin on the transcriptional activity of FOXO3, we next used RT-PCR to investigate the expression patterns of the anti-proliferative FOXO target genes Gadd45 (also known as Gadd45a), p27 $7^{k i p 1}$ (also known as Cdkn1b) and Bim (also known as Bcl2ll1; after stimulation with long- and normal-acting insulins (all methods performed as previously described) [5]. In line with our previous findings, we observed that treatment of FRTL-5 cells with normal insulin lowered levels of the cell cycle arrest inducer $p 27^{k i p 1}$ and the pro-apoptotic FOXO-target Bim in a dosage-dependent manner (Fig. 1b-d). Insulin glargine did not have a higher inhibitory potential on the transcriptional activity of FOXO3 in FRTL-5 cells as reflected by nonsignificant changes in FOXO target gene expression after treatment with insulin glargine compared with cells stimulated with normal insulin (Fig. 1b-d).

Similar to the lacking growth response, none of the insulins tested revealed significant alterations of mRNA expression of FOXO target genes in the FTC-133 cell line (data not shown). Finally, we wondered whether insulin glargine could activate the PI3K/AKT axis more potently than normal insulin. Therefore, the PI3K/AKT activation signature was studied after incubation with normal insulin and insulin glargine using Western blot analysis. FRTL-5 cells showed a dosage-dependent induction of AKT activation in response to insulin stimulation; however no significant difference could be observed between the two insulins studied (Fig. 1e). Being independent from exogenous insulin substitution, FTC-133 already featured high 
levels of AKT activation in combination with high levels of phosphorylated FOXO3 in insulin-deficient conditions, which could not be further amplified by subsequent stimulation with insulin or insulin glargine.

In summary, insulin glargine has identical effects on cellular proliferation in benign and malignant thyrocytes to those of normal insulin. Additionally, insulin glargine does not differentially affect the transcriptional activity of the tumour-suppressor FOXO3, which physiologically allows detection even of subtle changes in the PI3K/AKT activation status because of FOXO3's pivotal function in mitogenic PI3K/AKT signalling. However, we urgently emphasise that the observed effects are most likely to be cell-specific and are not suitable to universally rule out a mitogenic potency of insulin glargine or other insulin formulations.

Acknowledgements This study was supported by a DFG grant (FU 356/3-1) and an IZKF grant (B26).

Duality of interest The authors declare that there is no duality of interest associated with this manuscript.

\section{References}

1. Stumvoll M, Nawroth PP (2009) The insulin glargine dilemma: an opportunity for the diabetes community? Diabetologia 52:1987-1989

2. Jonasson JM, Ljung R, Talback M, Haglund B, Gudbjornsdottir S, Steineck G (2009) Insulin glargine use and short-term incidence of malignancies - a population-based follow-up study in Sweden. Diabetologia 52:1745-1754

3. Shukla A, Grisouard J, Ehemann V, Hermani A, Enzmann H, Mayer D (2009) Analysis of signaling pathways related to cell proliferation stimulated by insulin analogs in human mammary epithelial cell lines. Endocr Relat Cancer 16:429-441

4. Riesco-Eizaguirre G, Santisteban P (2007) Molecular biology of thyroid cancer initiation. Clin Transl Oncol 9:686-693

5. Karger S, Weidinger C, Krause K et al (2009) FOXO3a: a novel player in thyroid carcinogenesis? Endocr Relat Cancer 16:189-199

6. Weidinger C, Krause K, Klagge A, Karger S, Fuhrer D (2008) Forkhead box-O transcription factor: critical conductors of cancer's fate. Endocr Relat Cancer 15:917-929

7. Guigon CJ, Zhao L, Willingham MC, Cheng SY (2009) PTEN deficiency accelerates tumour progression in a mouse model of thyroid cancer. Oncogene 28:509-517

8. Weng LP, Gimm O, Kum JB et al (2001) Transient ectopic expression of PTEN in thyroid cancer cell lines induces cell cycle arrest and cell type-dependent cell death. Hum Mol Genet 10:251258 